\title{
Tsafon
}

Revue d'études juives du Nord

$78 \mid 2019$

L'antijudaïsme dans l'Antiquité

\section{Jérusalem, un statut en question}

\section{Emmanuel Persyn}

\section{(2) OpenEdition}

Journals

Édition électronique

URL : https://journals.openedition.org/tsafon/2522

DOI : $10.4000 /$ tsafon.2522

ISSN : 2609-6420

\section{Éditeur}

Association Jean-Marie Delmaire

\section{Édition imprimée}

Date de publication : 1 décembre 2019

Pagination : 125-144

ISSN : 1149-6630

\section{Référence électronique}

Emmanuel Persyn, « Jérusalem, un statut en question », Tsafon [En ligne], 78 | 2019, mis en ligne le 01 décembre 2019, consulté le 30 juin 2021. URL : http://journals.openedition.org/tsafon/2522 ; DOI : https://doi.org/10.4000/tsafon.2522 


\title{
Varia : Histoire
}

\section{Jérusalem, un statut en question}

\author{
Emmanuel Persyn*
}

Annoncée le 6 décembre 2017 par le président Donald Trump, la décision américaine de transférer l'ambassade des États-Unis de TelAviv à Jérusalem remet en cause une approche de la communauté internationale. Si elle ne constitue pas à proprement parler une surprise Donald Trump l'avait annoncée durant la campagne présidentielle et le Congrès a voté ce transfert le 9 mai 1995 -, elle compromet l'avenir des négociations israélo-palestiniennes, déjà réduites au strict minimum depuis quelques années.

Cet article traite des questions suivantes. Quelle est la perception de Jérusalem ? Quel est le statut juridique de cette ville revendiquée par les Israéliens comme par les Palestiniens? Quelle est la réalité économique, démographique et sociale sur le terrain?

Jérusalem est d'abord une ville sainte pour les trois religions monothéistes, juive, chrétienne et musulmane. "Quand un juif prie, il doit citer Jérusalem »: cet aphorisme montre l'importance de la ville dans le judaïsme. «Cité choisie par D., Jérusalem en vint à symboliser les valeurs et les aspirations les plus sublimes du judaïsme », note le Dictionnaire encyclopédique du Judaïsme ${ }^{1}$. Cité de David, elle devient la

\footnotetext{
${ }^{*}$ Université de Lille, Sciences Humaines et Sociales.

${ }^{1}$ « Jérusalem», Dictionnaire encyclopédique du judaïsme, Paris, éd. Cerf/Robert Laffont, coll. Bouquins, 1996, p. 516.
} 
capitale du royaume israélite unifié puis du seul royaume de Juda. Elle est surtout le centre religieux par excellence, la ville où est construit le Temple, lieu de la présence divine. Ce Temple est détruit à deux reprises : en 587 avant notre ère par Nabuchodonosor et en 70 de notre ère par le futur empereur romain Titus.

Dans la religion juive, le Messie doit reconstruire le Temple. Son retour marquera le rassemblement de tous les exilés, le rassemblement du genre humain et le monde à venir. En exil spirituel, tous les juifs reprennent depuis des temps immémoriaux le psaume de l'exilé à Babylone: «Si je t'oublie Jérusalem que ma droite se dessèche, que ma langue s'attache à mon palais si je ne me souviens pas de toi, si je ne place pas Jérusalem au sommet de mes joies » (Ps 137,5 et 6). Ils prient en direction de Jérusalem et la prière de la Amidah "contient un paragraphe entier où l'on implore $\mathrm{D}$. de revenir à Jérusalem, de reconstruire la ville et de rétablir la dynastie de David $»^{2}$. Durant l'année, trois jeûnes, dont celui de Tichah be-Av (9 Av), rappellent la destruction de la ville sainte, notamment du Temple. Chaque année, les fidèles achèvent la célébration de la Pâque, la fête qui rappelle la libération des Hébreux d'Égypte par le souhait «l'année prochaine à Jérusalem ».

Tout au long de l'histoire, quand ils y ont accès, les religieux juifs sont nombreux à s'installer en Palestine, notamment à Jérusalem, pour s'adonner à l'étude de la Torah et accomplir pleinement les mitsvot de la religion juive. «C'est l'aliya, la 'montée' vers la Terre d'Israël, considérée par les rabbins comme le premier des commandements de Dieu », écrit Élie Barnavi ${ }^{3}$. Ces juifs vivent dans un dénuement extrême, comptant le plus souvent sur les dons des communautés de la diaspora, et n'ont en tout état de cause aucune visée politique. Ils sont 25, 30000 « qui vivotent dans les quatre villes saintes : Jérusalem, Tibériade, Safed, Hébron ${ }^{4}$.

La deuxième communauté religieuse pour laquelle Jérusalem est une ville sainte est celle qui se réclame de Jésus-Christ. Pour les chrétiens, Jérusalem est avant tout le lieu de la passion du Christ, autrement dit de son arrestation, de sa crucifixion, de sa mort et de sa résurrection. Difficilement distingués des juifs au début puis persécutés

\footnotetext{
${ }^{2}$ Ibid.

${ }^{3}$ Élie Barnavi, Une histoire moderne d'Israël, Paris, éd. Flammarion, coll. Champs, 1988, p. 13.

${ }^{4}$ Ibid.
} 
par les empereurs romains ${ }^{5}$, les chrétiens sont ensuite tolérés. Après la conversion de l'empereur Constantin (280-337) en 312, le christianisme se propage, devient la seule religion d'État sous l'empereur Théodose le 8 novembre 392 et s'impose en Occident. Jérusalem devient lieu de pèlerinage à partir du $\mathrm{IV}^{\mathrm{e}}$ siècle. Les fidèles se mettent dans les pas du Christ et très tôt des monuments religieux sont construits sur les lieux présumés de la vie de Jésus : Cénacle, basilique du Saint-Sépulcre.

Confronté à des crises, le christianisme se divise en trois grandes branches, catholique, orthodoxe avec le schisme de 1054 et protestante avec les réformes de Martin Luther (1483-1546) et de Jean Calvin (15091564) au XVI ${ }^{\mathrm{e}}$ siècle, respectivement en $1517^{6}$ et 1536 . Au XIX ${ }^{\mathrm{e}}$ siècle, les religions chrétiennes défendent leurs positions et trouvent dans les puissances séculières des appuis. En soutenant les congrégations qui ouvrent des écoles, des hôpitaux et des institutions de bienfaisance, celles-ci assoient leur influence dans l'Empire ottoman qui ne peut s'y opposer. France, Angleterre, Russie, Allemagne et Autriche se livrent un combat diplomatique sans merci pour conforter leurs positions dans cette région du monde.

Enfin, depuis le $\mathrm{VII}^{\mathrm{e}}$ siècle, Jérusalem est une ville sainte de l'islam, comme le sont La Mecque et Médine : le prophète Muhammad (570 (?)-632) s'est élevé sur le coursier Buraq pour le voyage nocturne (isra') depuis le Rocher, sur lequel le calife 'Abd al-Malik (646-705) a construit la mosquée dite d'Omar. Jérusalem est sainte car elle a été « le théâtre d'un événement prophétique » puisqu' " elle a servi de point de départ pour le mi'raj, l'Ascension de Muhammad au ciel et qu'elle a eu ainsi un rôle fondamental dans la piété populaire et mystique $»^{7}$.

La visite nocturne constitue d'ailleurs la seule référence à Jérusalem que l'on trouve dans le Coran : "Gloire à celui qui a fait voyager de nuit son serviteur, de la mosquée sacrée (al-masjid al-haram à La Mecque) à la mosquée lointaine (al-masjid al-aqsa) afin de lui faire voir nos signes » (sourate XVII, verset 1). L'auteur poursuit en indiquant que «Jérusalem est liée à la révélation de l'un des grands piliers de l'islam, la prière ». D'ailleurs, au début de l'islam, les fidèles récitent leurs prières quotidiennes en se tournant vers Jérusalem. Selon un hadith rapporté par Boukhari (810-870), cela dure 16 à 17 mois.

\footnotetext{
${ }^{5}$ Notamment Néron en 74, Domitien en 93, Trajan en 106, Dèce en 250, Valérien en 257 et Dioclétien au début du IV ${ }^{\mathrm{e}}$ siècle.

${ }^{6}$ Martin Luther publie les 95 thèses le 31 octobre 1517 et il est excommunié en 1520.

${ }^{7}$ Toufic Fahd, article « Rites et fêtes », Dictionnaire de l'Islam, religion et civilisation, Encyclopaedia universalis, 1997, p. 738.
} 


\section{Une histoire mouvementée}

Ces perceptions religieuses de la ville sont à l'origine de son histoire mouvementée, même si elle ne suffit sans doute pas à en rendre totalement compte. Qu'elle soit appelée Yéroushalaïm ( La ville de la paix »), Jérusalem ou $\mathrm{Al}$ Quds ( La Sainte »), la cité sainte suscite en tout cas tous les engagements pour la défendre. Aussi bien, depuis sa création au troisième millénaire avant notre ère, a-t-elle été saccagée, détruite et occupée une trentaine de fois. «Selon l'archéologue israélien Meïr Ben Dov, du côté du Mont du Temple, la ville aurait été détruite et reconstruite vingt-cinq fois, de Salomon à Soliman », écrivent ainsi Alain Gresh et Dominique Vidal ${ }^{8}$. Elle fut successivement juive, égyptienne, babylonienne, romaine, byzantine, musulmane, chrétienne, arabe, ottomane, britannique...

Entre autres événements qui marquèrent son histoire, rappelons les plus dramatiques: la victoire du Babylonien Nabuchodonosor et la destruction du Temple de Salomon en 587 avant notre ère ; la destruction du second Temple par les Romains en 70 de notre ère ; la conquête de la ville par les armées musulmanes en 636 ; la reconquête chrétienne par les croisés de Godefroi de Bouillon en 1099 ; la reconquête arabe de Saladin en 1187 ; l'installation du pouvoir ottoman en 1522; l'expédition de Bonaparte en 1799 mais qui n'entre pas dans Jérusalem; l'occupation égyptienne en 1831; le retour des Ottomans en 1840; l'entrée des Britanniques en $1917 \ldots$

Venons-en maintenant à la question spécifique du statut de Jérusalem. Elle est relativement récente, compte tenu de l'émergence tardive d'une dimension internationale de la ville. «Délaissée par l'Europe depuis l'échec des Croisades, Jérusalem est à nouveau au centre des regards. Les voyageurs occidentaux se pressent autour des lieux saints qui prolifèrent comme jamais », écrit l'équipe dirigée par Vincent Lemire $^{9}$. À cet égard, le développement de la municipalisation de Jérusalem est « envisagé comme un moyen de lutter contre les tentatives d'ingérence des puissances consulaires ». La Grande-Bretagne ouvre un consulat en 1839, la Prusse en 1842, la France en 1843, l'Autriche en

\footnotetext{
${ }^{8}$ Alain Gresh, Dominique Vidal, Les 100 clés du Proche-Orient, « Jérusalem », Paris, Fayard, éd. Pluriel, $4^{\mathrm{e}}$ édition, 2011, p. 408.

${ }^{9}$ Vincent Lemire, (sous la dir. de, avec Katell Berthelot, Julien Loiseau et Yann Potin) Jérusalem. Histoire d'une ville-monde des origines à nos jours, Paris, éd. Flammarion, Coll. Champs, 2016, p. 319.
} 
1849, la Russie en 1847 par le biais de la Mission ecclésiastique de Jérusalem.

La question du statut de Jérusalem retient ensuite toute l'attention en raison notamment des Lieux saints. Avec cette première remarque de l'universitaire Monique Chemillier-Gendreau : «C'est la rivalité au sein des chrétiens, et notamment entre orthodoxes et catholiques ou entre États relevant de ces religions, qui a conduit au $\mathrm{XIX}^{\mathrm{e}}$ siècle certains Européens à avancer l'idée d'un statut particulier ${ }^{10}$. France, GrandeBretagne, Russie et même Allemagne se posent en États protecteurs des religions chrétiennes pour accroître leur influence auprès d'un Empire ottoman de plus en plus faible. Celui-ci s'efforce d'endiguer le mouvement. Ainsi, en 1867, il interdit au lieutenant britannique Charles Warren, envoyé par le Palestine Exploration Fund, de creuser sous l'esplanade des Mosquées. "Presque toutes les ruines à Jérusalem revêtent pour les musulmans un caractère sacré ", se désole l'archéologue qui tente alors de parvenir à ses fins par d'autres moyens, notamment le percement de tunnels et de puits ${ }^{11}$.

L'empire ottoman règle les conflits religieux récurrents en édictant le 8 février 1852 un firman qui reprend une décision de 1757. «Le statu quo détermine les sujets de la propriété des Lieux saints et plus concrètement les espaces à l'intérieur du sanctuaire ainsi que les horaires et la durée des fonctions, les déplacements, les parcours et la manière de les réaliser ", écrit la custodie, province de l'ordre franciscain, qui rappelle les communautés officiant au Saint-Sépulcre: latins, grecs, arméniens, coptes, syriens. En 1885, le traité de Berlin précise d'ailleurs : " Aucune atteinte ne saurait être apportée au statu quo dans les Lieux saints $»^{12}$. Ce statu quo perdure tant bien que mal jusqu'à la Première Guerre mondiale. L'engagement de l'Empire ottoman aux côtés des Empires centraux et sa défaite ont pour conséquence son démembrement et la tutelle européenne de ses anciennes provinces arabes. France et Grande-Bretagne se neutralisent autant qu'elles coopèrent pour asseoir leur domination sur cette région.

\footnotetext{
${ }^{10}$ Monique Chemillier-Gendreau, « Jérusalem : un avenir fondé sur les ressources du droit commun» dans Farouk Mardam-Bey, Élias Sanbar, Jérusalem, le sacré et le politique, Arles, éd. Sindbad Actes Sud, 2000, p. 311.

${ }^{11}$ Marius Schattner, « Archéologie et idéologie à Jérusalem », Esprit, $n^{\circ}$ 410, décembre 2014, à lire sur :

https://esprit.presse.fr/article/schattner-marius/archeologie-et-ideologie-a-jerusalem38168

${ }^{12}$ Alain Gresh, Dominique Vidal, op. cit., p. 409.
} 
La guerre n'est pas encore finie que Londres et Paris anticipent déjà le partage des provinces arabes de l'Empire ottoman en signant les accords Sykes-Picot le 16 mai 1916. Ils délimitent des zones d'administration directe, des zones d'influence et l'administration internationale de Jérusalem. Dans les négociations ultérieures qui aboutissent aux mandats délégués à la France et à la Grande-Bretagne par la Société des Nations le 24 juillet 1922, la question des Lieux saints et du statut de Jérusalem est âprement discutée et suivie avec attention par les autres pays. «Le prétexte de la protection des Lieux saints et la complicité des Églises chrétiennes sur ce sujet maintiennent les nations européennes, et particulièrement la France, dans l'idée qu'aucune solution ne doit conduire à la banalisation de Jérusalem et ne doit être exclusive d'un droit de regard des États chrétiens », analyse Monique Chemillier-Gendreau ${ }^{13}$.

Comme les Français, les Britanniques sont amenés à rendre régulièrement compte de leur administration à la SDN mais le mandat qu'ils ont sur la Palestine «ne comporte (en définitive) aucune administration internationale $»^{14}$. En 1920, 1921, 1929 et $1936^{15}$, les troubles éclatent à Jéricho, Jaffa ou Jérusalem et s'étendent ensuite au reste de Palestine. En 1929, les conditions d'accès au Mur occidental et la propriété des terrains qui l'entourent sont à l'origine des incidents qui dégénèrent en violents affrontements. Durant la période mandataire, la ville, qui se développe en dehors des murailles à partir de la fin du XIX siècle, continue de s'étendre. Les communautarismes religieux s'y développent, affectant même la municipalité. «On constate que dès le milieu des années 1920, les questions ne se traitent plus selon des critères techniques ou urbanistiques, mais dans le cadre d'une concurrence désormais explicite entre sionistes et nationalistes arabes ", écrit Vincent Lemire $^{16}$.

En 1937, la commission Peel ${ }^{17}$ envisage un premier partage de la Palestine, refusé aussi bien par les Juifs que par les Arabes. Présenté le 8 juillet 1937, ce plan prévoit une internationalisation de Jérusalem pour garantir l'accès aux lieux saints juifs, chrétiens et musulmans. Reprise par la commission Woodhead, cette hypothèse est abandonnée un an plus

\footnotetext{
${ }^{13}$ Monique Chemillier-Gendreau, op. cit., p. 312.

${ }^{14}$ Ibid., p. 313.

${ }^{15}$ En avril 1920, les deux jours d'affrontements lors du pèlerinage musulman de Nabi Musa, à proximité de Jéricho, font 6 morts de part et d'autre et une centaine de blessés.

${ }^{16}$ Vincent Lemire, op. cit., p. 372.

${ }^{17}$ Elle a été constituée par le gouvernement britannique en août 1936.
} 
tard, en novembre 1938, en raison de l'hostilité des protagonistes. Le Livre blanc du secrétaire d'État aux colonies Malcolm MacDonald (1901-1981), le 17 mai 1939, apparaît même comme une remise en cause de la politique suivie en restreignant considérablement l'immigration juive en Palestine - 15000 personnes par an pendant cinq ans - et en interdisant pour ainsi dire la vente de terres à des juifs. «La majorité arabe étant alors nettement acquise en Palestine (environ 1 million d'Arabes pour 500000 Juifs en 1939), le spectre de la partition s'éloigne, et miroite la garantie d'une indépendance prochaine dans le cadre d'un État à majorité arabe, avec Jérusalem pour capitale », écrivent ainsi Vincent Lemire, Katell Berthelot, Julien Loiseau et Yann Potin ${ }^{18}$.

\title{
Du partage à l'unification israélienne de Jérusalem
}

Après la Seconde Guerre mondiale, les Britanniques n'ont plus les moyens ni l'autorité pour administrer la Palestine comme ils le faisaient depuis qu'ils en avaient reçu le mandat de la Société des Nations en 1922. Confrontés aux revendications contradictoires des Arabes et des Juifs de Palestine, ils décident d'en référer à la nouvelle organisation internationale, l'Organisation des Nations Unies. L'ONU convoque donc une assemblée générale extraordinaire en 1947. Quelques mois plus tard, le 29 novembre 1947, elle adopte la résolution 181 qui décide le partage de la Palestine en deux États, juif et arabe. Elle prévoit également que Jérusalem sera un corpus separatum administré par les Nations Unies. La troisième partie de cette résolution consacrée à Jérusalem contient notamment 12 articles sur le statut de la ville. Elle est ainsi résumée par Alain Gresh et Dominique Vidal :

\begin{abstract}
Jérusalem devait constituer un "corpus separatum sous régime international spécial », démilitarisé, géré par un conseil de tutelle et un gouverneur ne pouvant être citoyen d'un des deux États prévus, garantissant les intérêts des Lieux saints des trois religions et la paix entre elles, inclus enfin dans l'union économique palestinienne. ${ }^{19}$
\end{abstract}

Walid Khalidi rapporte ce propos de Moshe Sharett (1894-1965), qui conduisait la délégation sioniste et qui souhaitait un corridor reliant Jérusalem à Tel-Aviv :

\footnotetext{
${ }^{18}$ Vincent Lemire, op. cit., p. 378.

${ }^{19}$ Alain Gresh, Dominique Vidal, op. cit., p. 409.
} 
Plusieurs États chrétiens parmi ceux approuvant le plan de partage avaient annoncé sans équivoque qu'ils ne voteraient ce plan que si Jérusalem obtenait un statut international. Il devint ainsi évident que la majorité requise pour le plan de partage ne pourrait être assurée si l'internationalisation de la Ville sainte n'était pas incluse dans la résolution. ${ }^{20}$

Entre autres États qui défendent cette position, il faut évidemment citer le Vatican qui se fait l'ardent défenseur de la protection des Lieux saints. Il refuse que les lieux saints chrétiens puissent être contrôlés par un État particulier. À la sainteté de la ville, à son universalité, Walid Khalidi ajoute une raison supplémentaire de faire de Jérusalem une ville internationale : la continuité territoriale de l'État arabe. «À partir de 1948, ce qu'on appelle la 'question de Jérusalem' ne porte plus, ou plus fondamentalement, sur la garde des Lieux saints, ni sur la liberté d'y accéder, mais sur le statut politique de la ville qui les abrite», remarquent Farouk Mardam-Bey et Élias Sanbar dans l'introduction de leur livre consacré à Jérusalem ${ }^{21}$.

Les faits ne correspondront pas à la résolution 181. Celle-ci est à peine votée que les affrontements reprennent en Palestine avec une violence accrue, d'abord entre Arabes et Juifs palestiniens puis, à partir du 15 mai 1948, entre Arabes et Israéliens. Durant cette guerre, Jérusalem est l'enjeu de combats acharnés. Les Israéliens expliquent les opérations engagées par la volonté de défendre les quartiers juifs. Walid Khalidi y voit l'application du plan Dalet: «L'objectif n'était rien de moins que la conquête et l'annexion de tout le corpus separatum, une zone où les propriétés juives ne dépassaient pas $6,6 \%$ du territoire $»^{22}$.

Lorsque cette première guerre se termine, il y a certes un État juif, mais pas d'État palestinien. La partie arabe est en effet administrée par deux États : la Cisjordanie par le royaume de Transjordanie et la bande de Gaza par l'Égypte. Jérusalem est divisée par une frontière. « La ligne d'armistice, dite 'Ligne verte', matérialise la séparation ${ }^{23}$. Le quartier juif de la vieille ville a été détruit ${ }^{24}$. La vieille ville, arabe, est administrée

\footnotetext{
${ }^{20}$ Walid Khalidi, « Pour une solution juste et viable » dans Farouk Mardam-Bey, Élias Sanbar, op. cit., p. 332.

${ }^{21}$ Farouk Mardam-Bey, Élias Sanbar, op.cit., p. 9.

${ }^{22}$ Ibid. p. 334.

${ }^{23} \mathrm{http}: / /$ ddc.arte.tv/cartes/234.

Page consultée le 19 janvier 2018. Le dessous des cartes, Arte.

24 «En réalité, avant 1948, la désignation de quartier 'juif” se référait aux locataires et non aux propriétaires. Seuls $20 \%$ du quartier juif d'avant 1948 dans la vieille ville appartenaient à des propriétaires juifs, alors que les $80 \%$ étaient des biens waqf
} 
par la Jordanie, la ville nouvelle, Jérusalem-Ouest, par le jeune État d'Israël. Dans le langage courant, on finit par parler de la «Vieille ville de Jérusalem », « Jérusalem-Est» pour désigner la partie jordanienne de la ville et de « Jérusalem » pour la partie israélienne.

L'ONU maintient néanmoins sa position en faveur d'une internationalisation de la ville. Elle l'indique dans les résolutions $194 \mathrm{du}$ 11 décembre 1948, 303 du 9 décembre 1949 et dans le projet de Conseil de tutelle présenté le 4 avril 1950. Cette disposition, contestée juridiquement par Monique Chemillier-Gendreau ${ }^{25}$, reste lettre morte. Aussi bien, note Frédéric Encel,

Cette solution ne présente au fond qu'une seule lacune : celle de ne recueillir aucun écho parmi les protagonistes sur le terrain. Les Israéliens ne céderont pas ce qu'ils possèdent déjà solidement et les Palestiniens ce qu'ils sont convaincus d'obtenir de gré ou de force à l'avenir. ${ }^{26}$

S'il garantit la liberté d'accès aux Lieux saints et la gestion des biens religieux par les autorités religieuses respectives, Israël fait de Jérusalem sa capitale le 23 janvier 1950, décision que la communauté internationale ne reconnaît pas en référence à la résolution 181 de l'ONU.

En juin 1967, au terme de la guerre des Six jours, l'armée israélienne occupe la Cisjordanie et la bande de Gaza. Elle investit la vieille ville, notamment l'ancien quartier juif et surtout le Mur occidental. Le quartier maghrébin qui le jouxte est évacué et rasé le 10 juin 1967 dans la soirée. L'esplanade est aménagée par une quinzaine d'entrepreneurs, le lendemain, à l'initiative du maire travailliste de la ville, Teddy Kollek (1911-2007) ${ }^{27}$. Quelques semaines plus tard, le 28 juin 1967, le gouvernement israélien élargit les frontières de la municipalité de Jérusalem qui passe, selon Walid Khalidi, de 6 à 73 $\mathrm{km}^{2}$ «aux dépens des territoires de la Cisjordanie occupée»: «En

appartenant à des familles chrétiennes et musulmanes de Jérusalem », écrit Walid Khalidi, op. cit., p. 337.

${ }^{25}$ Les États concernés n'avaient pas conclu d'accord à ce sujet comme cela avait été le cas pour Dantzig, Tanger, etc. «Le régime des mandats ne pouvait déboucher sur une autre issue, lorsqu'un mandat prenait fin, que celle du droit des peuples à disposer d'eux-mêmes. Et rien ne permettait de fragmenter le peuple pour le consulter. Il n'y avait donc pas lieu de doter les habitants de Jérusalem d'un statut différent de celui des Palestiniens », op. cit., p. 315.

${ }^{26}$ Frédéric Encel, Atlas géopolitique d'Israël, Aspects d'une démocratie en guerre, Paris, Éditions Autrement, 2008, p. 27.

${ }^{27}$ Il est maire de Jérusalem de 1965 à 1993. 
d'autres termes, écrit-il, une superficie supplémentaire venait d'être annexée par Israël en violation flagrante des conventions de Genève ${ }^{28}$. Présenté le 26 juillet 1967 mais révélé seulement en 1976, le plan du général Yigal Allon (1918-1980), vice-premier ministre israélien, prévoit l'agrandissement du couloir qui mène à Jérusalem. Kfar Etsion est reconstruit en 1967 avant d'autres implantations dans la vallée du Jourdain.

Sous la pression des religieux, notamment du Goush Emounim ${ }^{29}$, les implantations de civils sont d'abord tolérées puis agréées, voire encouragées par les autorités après la guerre de Kippour, dans les années soixante-dix. La première est créée à Ofra, au nord de Ramallah, en mai 1975, suivie de celle de Kfar Kaddoum, près de Naplouse, en novembre 1975. Dès lors, leur nombre ne cesse d'augmenter: selon les chiffres rapportés par Le Monde $e^{30}$, on dénombrait, en 2016, 131 colonies où vivent 399300 juifs $(4,67 \%$ de la population israélienne). Ces chiffres n'incluent pas Jérusalem-Est où la tendance est la même, voire accentuée. Au printemps 1968, au moment où le rabbin Moshe Levinger et ses partisans s'installent à Hébron, le gouvernement israélien confisque des terres à Jérusalem-Est pour «établir la liaison entre le mont Scopus et les quartiers juifs puis rétablir l'ancien quartier juif de la vieille ville », écrit Henry Laurens ${ }^{31}$ et l'esplanade est agrandie. Le 21 mai 1968, le Conseil de sécurité de l'ONU a d'ailleurs voté la résolution 252 qui «considère que toutes les mesures et dispositions législatives et administratives prises par Israël, y compris l'expropriation de terres et de biens immobiliers, qui tend à modifier le statut juridique de Jérusalem, sont non valides et ne peuvent modifier ce statut $»^{32}$.

La résolution 471 du Conseil de sécurité le 5 juin $1980^{33}$ et la déclaration du Conseil européen - neuf États réunis à Venise les 12 et 13 juin - ne disent pas autre chose :

\footnotetext{
${ }^{28}$ Walid Khalidi, op. cit., p. 336.

${ }^{29}$ « Bloc de la foi » créé en février 1974.

30 http://abonnes.lemonde.fr/les-decodeurs/article/2017/01/04/colonies-israeliennes-encisjordanie-pres-d-un-demi-siecle-d-installations-illegales_5057764_4355770.html Page consultée le 19 janvier 2018.

31 Henry Laurens, La question de Palestine, Le rameau d'olivier et le fusil du combattant, t. 4, Paris, éd. Fayard, 2011, p. 121.

${ }^{32}$ La résolution est adoptée par 13 voix et 2 abstentions, des États-Unis et du Canada. http://www.un.org/fr/documents/view_doc.asp?symbol=S/RES/252(1968)

Page consultée le 22 janvier 2018.

${ }^{33}$ Elle est votée par 14 voix contre 0, les États-Unis s'abstenant.
} 
Les Neuf reconnaissent le rôle particulièrement important que la question de Jérusalem revêt pour toutes les parties en cause. Les Neuf soulignent qu'ils n'acceptent aucune initiative unilatérale qui ait pour but de changer le statut de Jérusalem et que tout accord sur le statut de la ville devrait garantir le droit de libre accès pour tous aux Lieux saints. ${ }^{34}$

Le 30 juillet 1980, la Knesset vote néanmoins, par 69 voix contre 15, la Loi fondamentale qui indique que « Jérusalem entière et unifiée est la capitale d'Israël ${ }^{35}$. La communauté internationale rejette cette décision. Dans sa résolution 478 du 20 août 1980 votée par 14 voix contre 0 (abstention des USA), le Conseil de sécurité de l'ONU :

[...] affirme que l'adoption de la « loi fondamentale » par Israël constitue une violation $\mathrm{du}$ droit international [...] considère que toutes les mesures et dispositions législatives et administratives prises par Israël, la puissance occupante, qui ont modifié ou visent à modifier le caractère et le statut de la Ville sainte de Jérusalem, et en particulier la récente « loi fondamentale » sur Jérusalem, sont nulles et non avenues et doivent être rapportées immédiatement. ${ }^{36}$

D'ailleurs, les pays ont leur ambassade à Tel-Aviv et un consulat général à Jérusalem-Est ${ }^{37}$. Deux ans plus tard, le sommet arabe de Fès énonce dans son plan du même nom la revendication d' « un État palestinien indépendant ayant al-Qods pour capitale $»^{38}$. Cependant sur le terrain l'évolution amplifie la domination territoriale, économique et sociale israélienne.

Lorsque Israël et l'Organisation de Libération de la Palestine signent leur reconnaissance mutuelle les 9 et 10 septembre 1993, les espoirs sont grands même si l'on devine que certaines questions seront difficiles. Suffisamment difficiles pour les repousser à l'ultime phase des négociations sur le statut permanent. Ce sont notamment le droit au retour des réfugiés et le statut de Jérusalem. Dans un premier temps, le 13 septembre 1993, à Washington, Yitzhak Rabin, Premier ministre israélien, et Yasser Arafat, président de l'Organisation de Libération de la Palestine, signent, en présence du président américain Bill Clinton, la déclaration de principes portant sur les dispositions intérimaires

\footnotetext{
${ }^{34}$ Alain Gresh, Dominique Vidal, op. cit., p. 676 et sq.

${ }^{35}$ Le 14 décembre 1981, la Knesset adopte la loi d'annexion du plateau du Golan.

${ }^{36} \mathrm{http}$ ://www.un.org/fr/documents/view_doc.asp?symbol=S/RES/478(1980), page consultée le 18 janvier 2018.

3713 pays - Pays-Bas et 12 États sud-américains - ferment leur ambassade à Jérusalem.

${ }^{38}$ Alain Gresh, Dominique Vidal, op. cit., p. 678.
} 
d'autogouvernement. « La juridiction du Conseil s'étend au territoire de la Cisjordanie et de la bande de Gaza, à l'exception des points qui seront discutés dans le cadre des négociations sur le statut permanent: Jérusalem, les implantations, les emplacements militaires et les Israéliens », précise le document.

Les négociations sont plus que difficiles, constamment remises en question par les violences de ceux que l'on appelle les « ennemis de la paix » puis par des vagues d'attentats et des périodes de bouclage des territoires. Elles ne progressent que très, très lentement, jamais complètement sous le signe de la confiance mutuelle. Les deux camps se suspectent en permanence d'arrière-pensées, de duplicité, de non-respect des accords. Il faut constamment remettre le processus de paix sur les rails lors de sommets internationaux, le plus souvent sous tutelle américaine. Ce sont successivement Oslo I le 4 mai 1994, Oslo II le 28 septembre 1995, les accords de Wye Plantation le 23 octobre 1998, le sommet de Camp David du 11 au 24 juillet 2000.

En 1996, les négociateurs travaillistes proposent, lors de négociations secrètes :

[...] une solution en trois points : statut d'extra-territorialité et administration palestinienne pour l'esplanade des Mosquées, création d'une municipalité palestinienne gérant Jérusalem-Est (ainsi que les villages environnants) et reliée à la municipalité juive de Jérusalem-Ouest par un conseil municipal conjoint, mais maintien de la souveraineté israélienne. ${ }^{39}$

Ces négociations n'empêchent ni les tensions ni les conflits ; ainsi, 76 personnes meurent-elles du 27 au 29 septembre 1996 dans les affrontements provoqués par la décision de la municipalité juive de Jérusalem d'ouvrir un tunnel, pour des raisons archéologiques, en contrebas de l'esplanade des Mosquées. Puis, en 2000, est envisagée la possibilité d'un partage de souveraineté, piste de réflexion reprise à Taba du 21 au 27 janvier 2001. Cette rencontre «marquera le point le plus avancé des négociations entre les Palestiniens et l'équipe d'Ehoud Barak », notent Alain Gresh et Dominique Vidal. Ils ajoutent :

Les positions se rapprochent aussi sur le partage de souveraineté à JérusalemEst, selon le principe du plan Clinton : les quartiers arabes sont intégrés dans l'État palestinien, les quartiers juifs annexés par Israël. Les Palestiniens exigent

\footnotetext{
${ }^{39}$ Ibid, p. 412.
} 
la souveraineté sur le Haram El-Sharif (l'esplanade des Mosquées), les Israéliens sur l'ensemble du mur occidental. ${ }^{40}$

Mais après l'échec de Taba, la défaite d'Ehoud Barak et l'accession au pouvoir d'Ariel Sharon, la situation ne fait que se dégrader. En réalité, la région vit au rythme des attentats, des bouclages. Les années 20012004 sont particulièrement meurtrières. L'élaboration de la feuille de route par le Quartet (ONU, Union européenne, États-Unis et Russie) le 30 avril 2003, le sommet tripartite d'Akaba le 4 juin 2003 puis la rencontre Sharon-Abbas à Charm el-Cheikh, en Égypte, le 8 février 2005 ne donnent pas les résultats espérés. À partir de là, la conjoncture politique change du tout au tout, marquée notamment par la droitisation du gouvernement d'Israël et de son opinion publique, par la corruption, les luttes intestines et la radicalisation du mouvement palestinien.

\section{«Un grignotage lent et méthodique »}

Sur le terrain, l'évolution amplifie la domination territoriale, économique et sociale pour rendre irréversible ce statut décidé unilatéralement par Isräl. Depuis la guerre des Six jours, «les gouvernements successifs n'auront de cesse de modifier le visage de la ville, avec pour objectif principal d'y assurer une nette majorité juive », notent Alain Gresh et Dominique Vidal ${ }^{41}$. Destruction de quartiers arabes, expulsions, confiscation des biens immobiliers des absents ou des condamnés, expropriations par intérêt public, déclaration de parcs nationaux, ventes litigieuses... réduisent la présence palestinienne. En 1970, le gouvernement israélien exproprie 12300 dunams (1 230 hectares) pour construire des quartiers périphériques. La stratégie israélienne, telle que la décrit Alain Dieckhoff ${ }^{42}$, est de constituer « un anneau périphérique » en dehors des limites municipales, à une quinzaine de kilomètres puis de combler les vides à partir des années 1980. Ainsi Talpiot Est (Sud) ${ }^{43}$ et Ramot Allon (Nord) ${ }^{44}$ sont-ils planifiés dès 1971 ,

\footnotetext{
${ }^{40} \mathrm{Ibid}, \mathrm{p} .165$.

${ }^{41}$ Ibid, p. 410.

${ }^{42}$ Cité par Vicncent Lemire, op. cit., p. 410. Alain Dieckhoff, Les espaces d'Israël. Essai sur la stratégie territoriale israélienne, Paris, Presses de la fondation nationale des sciences politiques, 1989.

${ }^{43}$ Construit en 1973, ce quartier comptait 15398 habitants en 2015.

${ }^{44}$ Ce nouveau quartier est inauguré en 1973, selon Frédéric Encel, comme les autres. Ce quartier compte quelque 50000 habitants en 2010, selon Y Net. https://www.ynetnews.com/articles/0,7340,L-3954217,00.html
} 
Gilo (Sud-Ouest) et Neveh Ya'acov (Nord) l'année suivante ${ }^{45}$. Dans le nord, Pisgat Zeev est un «satellite» programmé en 1982 dans le but d'assurer la liaison entre le quartier de French Hill et de Neveh Ya'acov.

En 1991, les premiers colons s'installent dans le quartier arabe de Silwan, à quelques mètres de la mosquée El-Aqsa, en face du Mont des Oliviers. Vingt-six ans plus tard, «nous ne sommes plus que trois dans ce coin », raconte une Palestinienne à Claude Guibal, grand reporter de France Inter ${ }^{46}$. Ils sont aujourd'hui plusieurs centaines d'Israéliens à habiter dans ce quartier, proche de la Cité de David, dont 500 religieux juifs selon Piotr Smolar, correspondant du Monde en Israël.

C'est de cette année que date la première intervention immobilière d'Elad, acronyme de l'expression hébraïque «vers la cité de David » qui aide les familles juives à s'installer à Jérusalem-Est pour constituer une majorité juive dans les quartiers arabes de la ville. Créée en 1986 par David Bééri, cette fondation controversée mais très riche mène des actions en justice pour la restitution de maisons à des propriétaires juifs, rachète des biens dont les propriétaires sont déclarés absents, fait pression sur les Palestiniens pour accepter de vendre leur maison ou encore construit des logements. Selon Marius Schattner :

Elad juge plus utile de procéder à des achats de terrains ou de maisons, quitte à y mettre le prix fort. Les transactions à vrai dire plutôt rares s'opèrent par le biais de sociétés écran, dans des conditions souvent douteuses, les résidents palestiniens concernés se plaignant d'avoir été bernés ou même d'avoir été victimes de faux en écritures. Mais il faut savoir que la vente de biens fonciers à des Israéliens est passible de mort selon les lois de l'Autorité palestinienne et de la Jordanie. $^{47}$

Le constat reste d'actualité à en juger d'après le témoignage publié par Nir Hasson dans Ha'aretz le 5 janvier 2018. Autre organisation très controversée, Ateret Cohanim (« La couronne des grands prêtres »), une association de colons religieux et ultra-nationalistes fondée aux États-

\footnotetext{
Page consultée le 25 janvier 2018.

${ }^{45} \mathrm{https}$ ://jerusalem-info.com/pisgat-zeev/

Page consultée le 23 janvier 2018.

$46 \mathrm{https} / /$ www.franceinter.fr/emissions/le-zoom-de-la-redaction/le-zoom-de-laredaction-13-decembre-2017

Page consultée le 19 janvier 2018.

${ }^{47}$ Marius Schattner, « Archéologie et idéologie à Jérusalem », Esprit, n 410, décembre 2014, consulté sur https://esprit.presse.fr/article/schattner-marius/archeologie-et-ideologie-a-jerusalem38168.
} 
Unis en 1978 et « dont l'objectif est la construction du troisième Temple en lieu et place de la mosquée du Rocher». Elle est présidée par Matitiyahou Dan, «depuis les années 1980, l'un des acteurs clés dans l'acquisition de propriétés palestiniennes destinées à l'implantation juive à Jérusalem-Est $\gg{ }^{48}$.

Tous les moyens sont bons, raconte le journaliste: prestations sexuelles, voyages, sociétés écrans, chantage, etc. L'association a récemment été mise en cause par le patriarche de Jérusalem de l'église grecque orthodoxe, Théophile III, dans une lettre ouverte publiée par le Guardian: "L'une des menaces les plus importantes pour les chrétiens en Terre sainte est l'activité inacceptable des groupes pro-implantations radicaux qui tentent de prendre le contrôle des biens aux alentours de la porte de Jaffa $»^{49}$. Cette Église ${ }^{50}$ est, en fait, confrontée à la corruption du patriarche Irineos $1^{\mathrm{er}}$, destitué en 2005.

Rachat de maisons, installations individuelles, programmes collectifs... Il serait fastidieux d'établir la chronologie détaillée de la pénétration juive dans Jérusalem-Est. Betselem, le Centre israélien d'information sur les droits de l'homme dans les territoires occupés, la résume ainsi :

Quinze grands quartiers israéliens ont été construits dans des zones qu'Israël a annexées à la municipalité de Jérusalem après 1967. Ces quartiers sont également considérés comme des colonies de droit international. En outre, des enclaves de colons ont été construites au cœur des quartiers palestiniens dans ces zones annexées, avec l'aide du gouvernement israélien et de la municipalité de Jérusalem. $^{51}$

En 1992, les terres privées de Har Homa, entre Jérusalem et Bethléem, sont expropriées. Le 25 février 1997, le gouvernement Netanyahou annonce son projet de création d'une colonie juive, Har Homa, dans la partie arabe occupée de Jérusalem, « ce qui isolerait un

\footnotetext{
${ }^{48}$ Nir Hasson, Ha'aretz, 4 janvier 2018, article traduit dans Courrier international, $\mathrm{n}^{\circ} 420,18$ janvier 2018.

${ }^{49}$ Stuart Winer, "Théophile III dénonce 'les militants pro-implantations radicaux' », The times of Israël, 8 janvier 2018. https://fr.timesofisrael.com/theophile-iii-denonceles-militants-pro-implantations-radicaux-

Page consultée le 19 janvier 2018.

${ }^{50}$ Cette Église compte 220000 fidèles en Jordanie, Cisjordanie, Israël et dans la bande de Gaza. Elle est la plus nombreuse et la plus riche des Églises chrétiennes de Palestine.

${ }^{51} \mathrm{https}$ //www.btselem.org/settlements/statistics

Page consultée le 21 janvier 2018.
} 
peu plus la ville sainte de son environnement arabe $»^{52}$. Il prévoit la construction de 6500 logements réservés aux juifs. La construction commence le 28 mars suivant. Réunie en session spéciale le 15 juillet 1997, l'Assemblée générale de l'ONU condamne par 131 voix contre 3 (Israël, États-Unis et Micronésie) et 10 abstentions cette construction et plus largement la colonisation des territoires occupés. Le 9 septembre 1997, en tournée dans la région pour renouer le dialogue, la Secrétaire d'État américaine Madeleine Albright condamne à son tour. En 2003, Ariel Sharon autorise la construction de 91 logements à Jabal Moukaber, ensemble qui devient Nof Zion.

Le 17 novembre 2009, soumis à la pression américaine, le gouvernement suspend la construction de nouveaux logements en Cisjordanie pour dix mois. Cependant Jérusalem n'est pas concernée par cette mesure et la construction des 3000 logements en cours est poursuivie. La crise rebondit quelques mois plus tard. Le 10 mars 2010, Mahmoud Abbas annonce la rupture des négociations avec Israël après l'annonce d'un programme de construction de 1600 logements à Jérusalem. Relancée par l'émissaire américain George Mitchell, ces négociations, indirectes, sont de nouveau interrompues à la fin du moratoire israélien sur la colonisation. Le 8 novembre 2010, ce sont 1300 logements à Har Homa, dans la Jérusalem arabe. Le 11 août 2013, Uri Ariel, ministre du logement du gouvernement Netanyahou, annonce un programme de 793 unités à Jérusalem-Est ${ }^{53}$. Le 25 octobre 2016, le gouvernement a encore approuvé la construction de 176 logements à Nof Zion, qui en compte déjà 91 , dans le quartier de Jabal Moukaber, un village intégré dans le territoire de la municipalité en $1967^{54}$. Le journaliste précise que «cela en ferait la plus importante implantation juive au sein d'un quartier palestinien de Jérusalem ».

La qualité de résident, qui confère moins de droits que la citoyenneté $^{55}$, n'est plus octroyée qu'au compte-gouttes aux Palestiniens

\footnotetext{
${ }^{52}$ Henry Laurens, La question de Palestine, La paix impossible, t. 5, Paris, éd. Fayard, 2015 , p. 596.

${ }^{53}$ Hervé Amiot, « Histoire d'une colonisation depuis 1967 », article sur https://www.lesclesdumoyenorient.com/Les-implantations-israeliennes-en-Cisjordanie2-histoire-d-une-colonisation.html, page consultée le 19 janvier 2018.

54 http://www.rfi.fr/moyen-orient/20171026-jerusalem-est-nof-zion-construction-jabalmoukaber-quartier-palestinien

Page consultée le 18 janvier 2018.

55 "La résidence permanente confère moins de droits que la citoyenneté. Il permet à son titulaire de vivre et de travailler en Israël et de recevoir des prestations sociales en vertu
} 
habitant Jérusalem et peut être très facilement supprimée. L'association Betselem note ainsi, le 3 avril 2017, que « depuis 1967, Israël a révoqué la résidence permanente de quelque 14500 Palestiniens de JérusalemEst ${ }^{56}$. Le gouvernement poursuit un objectif : expulser les Palestiniens des zones que l'État d'Israël veut absolument annexer et les envoyer audelà du mur de séparation construit de 2002 à 2016. Ce mur suit d'ailleurs un tracé qui ne correspond pas à la ligne verte mais qui permet l'intégration des colonies et supprime au contraire toute continuité territoriale d'un éventuel État palestinien. Betselem écrit encore :

Les nouvelles limites municipales de Jérusalem, s'inspiraient en grande partie des préoccupations démographiques, la principale d'entre elles étant d'exclure les zones palestiniennes densément peuplées afin d'assurer une majorité juive à Jérusalem. Conformément à cette logique, Israël a inclus certaines terres appartenant à des villages près de Jérusalem dans la juridiction municipale de la ville, tout en laissant les propriétaires à l'extérieur. Cela s'est produit, par exemple, avec Beit Iksa et al-Birah au nord, et avec des zones faiblement peuplées dans les juridictions municipales de Bethléem et de Beit Sahour au sud. Ce faisant, Israël a divisé les villages et les quartiers palestiniens, n'en annexant que des parties ${ }^{57}$.

Les quartiers arabes de Jérusalem, écrit Piotr Smolar ${ }^{58}$, «sont restés une sorte de trou noir sans rattachement véritable»: «Leurs habitants n'ont partagé ni le développement de Jérusalem-Ouest ni le destin cahoteux de la Cisjordanie ». Les disparités entre les deux communautés sont énormes. Ainsi, les Palestiniens habitant Jérusalem paient-ils des impôts locaux comme les autres mais ne bénéficient pas des mêmes services publics, qu'il s'agisse des transports, du ramassage des ordures ménagères, des structures de santé, des écoles ou de l'aménagement urbain. Développement anarchique, empilement de

de la loi sur l'assurance nationale, ainsi que de l'assurance maladie. Mais les résidents permanents ne peuvent pas participer aux élections nationales - soit en tant qu'électeurs ou en tant que candidats - et ne peuvent pas se présenter aux élections, bien qu'ils aient le droit de voter aux élections locales et de se présenter aux élections municipales », Betselem.

${ }^{56}$ https://www.btselem.org/jerusalem

Page consultée le 21 janvier 2018.

57 Ibid.

${ }^{58}$ Piotr Smolar, « Les emmurés de Jérusalem-Est », Le Monde, 29 mai 2017. http://abonnes.lemonde.fr/proche-orient/article/2017/05/30/les-emmures-de-jerusalemest_5135752_3218.html

Page consultée le 19 janvier 2018. 
constructions arabes, ce qui reste de la Silwan arabe « pourrit sur pied », selon le journaliste. Il cite ce témoignage d'un habitant :

Aujourd'hui, les gamins de Silwan n'ont rien à faire et nulle part où aller. Et pendant ce temps, dans les quartiers juifs, il y a des espaces verts, des centres commerciaux, une vie agréable. Depuis 1967, on vit dans le mensonge, celui de négociations n'aboutissant à rien. Et pendant ce temps, El Ad veut judaïser Silwan et jeter les Palestiniens dehors.

S'ils ne payaient pas d'impôts, les Palestiniens seraient passibles d'expropriation...

La situation des habitants palestiniens se dégrade constamment : de 15 à 20000 logements construits sans permis jusqu'en 2004, - « de 2004 à fin septembre 2017, les autorités israéliennes ont démoli 730 logements unités à Jérusalem-Est » - une densité double de celle des quartiers juifs en l'absence de réserves foncières « pour la population palestinienne qui a plus que quintuplé depuis 1967 » (1,9 personne par chambre pour 1), note encore Betselem. En résumé, remarque Piotr Smolar, « un grignotage, lent et méthodique, a été organisé par les colons, avec le soutien des autorités $»^{59}$. Dans son émission « Le dessous des cartes ${ }^{60}$, Arte prend l'exemple de la desserte du tramway de Jérusalem et de l'implantation des parcs relais à proximité des stations. La ligne doit desservir les quartiers arabes et le nord. Douze des 23 stations implantées «sont soit sur la ligne verte soit au-delà, c'est-à-dire dans Jérusalem Est ». Et de remarquer : « Pour les opposants, le tramway serait la énième manifestation de l'emprise israélienne sur Jérusalem-Est, en reliant les habitants des implantations à l'ouest en violation du droit international ».

Le 23 décembre 2016, le Conseil de sécurité de l'ONU vote une nouvelle fois une résolution, la 2334, condamnant la colonisation de Jérusalem-Est et de la Cisjordanie.

Il réaffirme que la création par Israël de colonies de peuplement dans le Territoire palestinien occupé depuis 1967, y compris Jérusalem-Est, n'a aucun fondement en droit et constitue une violation flagrante du droit international et un obstacle majeur à la réalisation de la solution des deux États et à l'instauration d'une paix globale, juste et durable. ${ }^{61}$

\footnotetext{
${ }^{59}$ Ibid.

${ }^{60}$ « Un tramway à Jérusalem », émission diffusée le 3 avril 2012.

${ }^{61} \mathrm{http}$ ://www.un.org/fr/documents/view_doc.asp?symbol=S/RES/2334(2016)

Page consultée le 21 janvier 2018.
} 


\section{Ce n'est pas la position d'Israël comme l'explique Le Figaro :}

La position de la communauté internationale découle principalement de la $4^{\mathrm{e}}$ Convention de Genève, dont l'article 49-6 interdit à la puissance occupante « de déporter ou de transférer une partie de sa population civile dans les territoires qu'elle occupe ». Mais l'État juif soutient pour sa part que ce texte ne s'applique pas à la Cisjordanie dans la mesure où aucune souveraineté n'y était reconnue par la communauté internationale avant la guerre des Six-jours, et moins encore à Jérusalem-Est puisque celle-ci fut annexée unilatéralement le 28 juin $1967{ }^{62}$

Ce nouveau rappel de la communauté internationale - la résolution a été votée par 14 des 15 membres du Conseil de sécurité - reste, elle aussi, sans effet puisque la colonisation de Jérusalem-Est est relancée dès le mois suivant avec l'annonce, le dimanche 22 janvier 2017, de l'extension de trois quartiers juifs (Pisgat Zeev, Ramot et Ramat Shlomo) où la construction de 566 logements est validée et de la programmation de 11000 logements supplémentaires. Depuis l'investiture de Donald Trump le vendredi 20 janvier 2017, le gouvernement israélien «a procédé à cinq annonces d'extension de colonies portant sur plus de 6000 logements en Cisjordanie et à Jérusalem-Est occupées », note l'AFP le 31 mars 1917.

Pour conclure, on peut résumer cette emprise israélienne croissante sur Jérusalem-Est par quelques chiffres.

- La superficie du territoire municipal israélien est passé de $6 \mathrm{~km}^{2}$ en 1947 à $71 \mathrm{~km}^{2}$ (73 selon d'autres) en 1967 et à $164 \mathrm{~km}^{2}$ en 2002, dont $24,5 \mathrm{~km}^{2}$ expropriés selon Betselem.

- La ville de Jérusalem compte 882700 habitants en 2016 selon le bureau central des statistiques israélien, dont 300000 Palestiniens.

- La population juive, qui dans tout le pays décuple depuis 1947, passe de 2400 personnes en 1947 à plus de 200 000, dont plusieurs centaines à Silwan $^{63}$. Selon l'association Ir Amim, le nombre d'Israéliens habitant Jérusalem-Est a augmenté de $20 \%$ de 2000 à 2012.

Je citerai également ce point de vue de l'équipe de Vincent Lemire :

\footnotetext{
62 http://www.lefigaro.fr/international/2016/12/24/01003-20161224ARTFIG00066-lescolonies-israeliennes-50-ans-d-expansion-et-de-polemiques.php Page consultée le 21 janvier 2018.

${ }^{63}$ Henry Laurens, "Jérusalem, capitale de la Palestine mandataire » dans Jérusalem, Le sacré et le politique, op. cit., p. 226.
} 
Si le mur de séparation construit par les autorités israéliennes depuis 2002 n'isolait pas Jérusalem des villes voisines palestiniennes, la Ville sainte entrerait en conurbation avec les agglomérations de Ramallah au nord et de Bethléem au sud, au sein d'une aire urbaine effective d'environ $400 \mathrm{~km}^{2}$ si on y ajoute les blocs de colonies situés au nord (Givat Zeev), au sud (Gush Etzion) et à 1'Est (Maale adumim). ${ }^{64}$

Ce constat anticipe l'échelle pertinente à laquelle il faudra sans doute gérer les problèmes urbains de demain.

Ainsi la décision américaine peut être interprétée comme la prise en compte de la réalité du terrain. C'est d'ailleurs l'argument que fait valoir la Maison Blanche. Elle n'en est pas moins un engagement supplémentaire en faveur d'Israël, une remise en cause du droit international et un obstacle à la négociation qui devra bien se tenir un jour ou l'autre, dans le cadre de deux États ou d'un seul. Une négociation qui semble pour le moment devoir rester dans l'impasse après la décision du parlement israélien. Celui-ci a en effet adopté le mardi 2 janvier 2018 un projet de loi qui prévoit qu' « il ne faudra donc plus 61 mais 80 députés (sur 120) pour approuver un transfert vers une souveraineté palestinienne, dans le cadre d'un futur accord de paix $»{ }^{65}$.

\footnotetext{
${ }^{64}$ Vincent Lemire, op. cit. p. 356.

${ }^{65}$ Claire Bastier, "Israël vote une loi pour empêcher la division de Jérusalem », Le Monde.fr, 3 janvier 2018.

https:/www.lemonde.fr/proche-orient/article/2018/01/03/israel-vote-une-loi-pourempecher-la-division-de-jerusalem_5236955_3218.html
} 